\title{
Application of Dried Human Amnion Graft to Improve Post-Prostatectomy Incontinence and Potency: A Randomized Exploration Study Protocol
}

\author{
Dimitri Barski (1) - Holger Gerullis · Thorsten Ecke $\cdot$ Mihaly Boros • \\ Jan Brune · Ulrich Beutner · Igor Tsaur · Albert Ramon • \\ Thomas Otto
}

Received: September 24, 2019 / Published online: November 28, 2019

(c) The Author(s) 2019

\section{ABSTRACT}

Introduction: Incontinence (up to 20\%) and erectile dysfunction (up to 70\%) occur frequently after radical prostatectomy (RP) in patients with localized prostate cancer. Human amniotic membrane (HAM) can improve tissue regeneration and functional outcome after RP owing to the growth factors and unique immune tolerance. Preliminary studies showed the potential

Enhanced Digital Features To view enhanced digital features for this article go to https://doi.org/10.6084/ m9.figshare.10283555.

D. Barski $(\bowtie) \cdot$ T. Otto

Department of Urology, Rhineland Clinic, Lukas

Hospital Neuss, Preussenstr. 84, 41464 Neuss,

Germany

e-mail: dimitri.barski@rheinlandklinikum.de

H. Gerullis

University Hospital for Urology, Klinikum

Oldenburg, School of Medicine and Health Sciences,

Carl von Ossietzky University, Oldenburg, Germany

T. Ecke

Department of Urology, HELIOS Hospital, Bad

Saarow, Germany

M. Boros

Department of Experimental Surgery, University of

Szeged, Szeged, Hungary

J. Brune

DIZG, Deutsches Institut für Zell- und Gewebeersatz

gGmbH, Berlin, Germany value of HAM in the reconstruction of the urinary tract and nerve protection during RP.

Methods: A protocol is developed for a prospective, randomized, single-blind, singlesurgeon, placebo-controlled exploration study of the efficacy and safety of dehydrated human amnion membrane placed around the neurovascular bundle (NVB) and vesicourethral anastomosis (VUA) during RP for the treatment of localized prostate cancer. Eligible for inclusion are patients with localized prostate cancer, requiring a surgical procedure and exclusion of preoperative incontinence and erectile dysfunction. The patients are randomized 1:1 to HAM vs. placebo and blinded during the study

U. Beutner

Department of General, Visceral, Endocrine and Transplantation Surgery, Kantonsspital St. Gallen, St. Gallen, Switzerland

I. Tsaur

Department for Urology and Pediatric Urology, University Medical Center of Johannes Gutenberg, Mainz, Germany

\section{A. Ramon}

International Tissue Engineering Research

Association (ITERA), Antwerp, Belgium

T. Otto

University of Duisburg-Essen, Essen, Germany 
period. According to the $T$ test with an alpha of 0.05 and a power of $80 \%$ and expecting a dropout of $20 \%$ of the patients, an adjusted sample size per arm of 164 patients is required. Planned Outcomes: The primary outcome is a postoperative continence measured as 24 -h pad test up to 12 months postoperatively. Secondary outcomes are potency, time of postoperative catheter removal, postoperative complications, and biochemical recurrence. The protocol for this randomized exploration study defines the conditions to assess the efficacy and safety of HAM application during RP in order to improve the postoperative functional outcome. This trial should pave the way for future studies of tissue engineering in an effort to reduce the morbidity of RP. Trial Registration: Clinicaltrials.gov, identifier NCT03864939.

Keywords: Amniotic membrane; Erectile dysfunction; IDEAL recommendations; Incontinence; Prostatectomy; Surgical innovation; Urology

\section{Key Summary Points}

Why carry out this study?

High prevalence of incontinence (up to $20 \%$ ) and erectile dysfunction (up to 70\%) after radical prostatectomy (RP)

There is an unmet need to improve tissue regeneration; current technical innovations of RP have not proven to be effective

Human amniotic membrane (HAM) can improve tissue regeneration and functional outcome after RP owing to the growth factors and unique immune tolerance, as shown in preliminary studies

What will be learned from the study?

If there will be a change to an early postoperative continence, potency, and complications by application of human amniotic grafts during RP, when compared with standard surgery in a randomized exploration study

\section{INTRODUCTION}

Functional outcome is an important parameter of quality of life after radical prostatectomy (RP) in prostate cancer. Incidence of post-prostatectomy incontinence (PPI) varies greatly depending on the surgical technique, expertise, and patient factors $[1,2]$. The postoperative continence rate (no pads) varies between $4 \%$ and $31 \%$, mean $16 \%$ [2]. Some patients recover from incontinence after rehabilitation, but $10-20 \%$ suffer from persistent incontinence and 20-70\% from erectile dysfunction $[1,3,4]$. In addition, continence is not standardized and is defined differently in studies: 1 -h or 24 -h pad weighing test, pad consumption, social continence [5].

The anatomical understanding and surgical techniques during RP have been significantly improved over the last decade, as well as technical innovations with $\times 10$ magnification, 3D viewing, and instrument miniaturization. However, open retropubic radical prostatectomy remains standard surgical therapy for localized prostate cancer. Laparoscopic and robotic assisted RP have not shown any benefits in terms of oncological and functional outcomes compared to open RP [6-8]. New techniques with nerve-sparing principles of the neurovascular bundle have so far not significantly improved the functional outcome.

Surgical intervention is the main reason for scarring and nerve damage [9]. The damage of the plexus in the dorsal prostatic capsule occurs because of mobilization and traction of the tissue. In addition, a local inflammatory response is triggered, which causes the delay to reaching continence and potency. The inflammatory response triggers edema, acidosis, and apoptosis, which can potentially lead to nerve damage $[10,11]$. So far, there is no ideal strategy for preventing scar and adhesion formation in the nervous system. Clinical benefit of growth and anti-inflammatory factors could be crucial for the regeneration of the neurovascular bundle (NVB) [11].

Grafts of human amniotic membrane (HAM) could be a solution. They are available, flexible, robust, and offer unique anti-inflammatory and anti-fibrotic properties that allow optimal 
regeneration [12]. HAM contains cytokines, growth factors, and neurotrophic factors that slow down the inflammatory response $[13,14]$. Mesenchymal amniotic cells have stem cell-like properties [15]. HAM has been used for decades for corneal reconstruction and treatment of complicated wound defects $[16,17]$. Amnion is thus a promising biomaterial for regenerative medicine and is excellently suited for use in the urinary tract, as shown in our own experimental and clinical work [18-21]. New data show the prevention of nerve fibrosis by amniotic wounds in animal experiments [14]. However, the current level of evidence is low because of the few experimental and retrospective studies available.

This manuscript describes the design of a randomized, single-blind, placebo-controlled, phase 2 study of the efficacy and safety of HAMassisted radical prostatectomy.

\section{METHODS}

\section{IDEAL Recommendations}

The presented method of HAM application during RP is evaluated according to the IDEAL recommendations of surgical innovation. The introduction and description of new surgical methods, innovations, or variations does not yet follow clear standardized paradigms as is the case with the development process of new pharmaceutical agents and drugs. Innovation, Development, Exploration, Assessment and Long-term Study (IDEAL) is a reporting approach, introduced in 2009 by an international panel of surgeons, researchers, journal editors, methodologists, statisticians, and other stakeholders who are committed to producing, disseminating, and evaluating quality research in surgery [22]. Recently, IDEAL stages were adopted to evaluate and regulate the use of medical devices (IDEAL-D) as well (Table 1) [23]. IDEAL provides clear stages for surgical innovation which make it possible to assign all research to its particular level of development and evidence [23]. This approach is unique and paves the way for an efficient, actionable, and transparent quality improvement system for the life cycle of development and post-market monitoring of new medical devices and implants.

\section{Surgical Procedure}

$\mathrm{RP}$ is performed by one surgeon (TO) with an individual experience of more than 1000 surgeries of this kind. The surgical procedure is retropubic prostatectomy according to the technique of Walsh [24]. The standard surgical steps are not influenced by the clinical trial. For low and medium risk prostate carcinoma according to D'Amico classification, localized prostate cancer, and preoperative potency with International Index of Erectile Function (IIEF$5) \geq 20$, uni- or bilateral nerve preservation takes place by preservation of the seminal vesicles and neurovascular bundles [6, 25]. Dehydrated amniotic membrane (DIZG, gGmbH, Germany) is divided into three pieces $(3 \times 1 \mathrm{~cm})$. Initially, the NVB is overlayed with amnion after reconstruction of Denonvillier's fascia. Subsequently, the vesicourethral anastomosis (VUA) is prepared and then wrapped with HAM (Fig. 1) [26].

\section{Amniotic Membrane}

The dried HAM (German Institute for Cell and Tissue Replacement, DIZG gGmbH, Germany) has been licensed since 22 December 2015 by the Paul-Ehrlich-Institut (PEI.H.03357.01.1) for implantation in humans for temporary skin replacement in thermal injuries and for the corneal surface repair and other surgical reconstructive techniques. HAM is obtained by donation as part of a caesarean section, sterilized, and made ready for use. Each donor is screened for exclusion based on the history and physical examination. Infectious serology tests and tests for viral infections by nucleic acid amplification (NAT) follow. The screening currently includes tests for antibodies to hepatitis A, B, C and HIV-1/2 as well as cytomegalovirus (CMV), Treponema pallidum, and human T-lymphotrophic virus $1 / 2$ (HTLV$1 / 2$ ), NAT tests for hepatitis B, C and HIV genomes. Despite these extensive investigations, 
Table 1 Stages of the IDEAL-D framework [23]

\begin{tabular}{|c|c|c|c|c|}
\hline & & Primary outcome & Study design & Patients \\
\hline Stage 0 & Preclinical & Concept, safety & $\begin{array}{l}\text { Experimental studies } \\
\text { (animal, cadaver) }\end{array}$ & \\
\hline Stage 1: Idea & "First in human" & Innovation & $\begin{array}{l}\text { Case report, case } \\
\text { series, registration }\end{array}$ & $\begin{array}{l}\text { Single to } \\
\text { few } \\
(<10)\end{array}$ \\
\hline $\begin{array}{l}\text { Stage 2: Development } \\
\text { and exploration }\end{array}$ & $\begin{array}{l}\text { “Tinkering” with } \\
\text { device, few adopters }\end{array}$ & Development, safety, efficacy & $\begin{array}{l}\text { Prospective cohort } \\
\text { trials }\end{array}$ & $10-100$ \\
\hline Stage 3: Assessment & Stable procedure & $\begin{array}{l}\text { Compare to standard, clinical } \\
\text { outcome }\end{array}$ & $\begin{array}{l}\text { Randomized } \\
\text { controlled trial or } \\
\text { similar }\end{array}$ & $>100$ \\
\hline Stage 4: Long term & $\begin{array}{l}\text { Registry, long-term } \\
\text { evaluation }\end{array}$ & $\begin{array}{l}\text { Quality assurance, identification of } \\
\text { risk factors, comparators }\end{array}$ & Registry & $>100$ \\
\hline
\end{tabular}

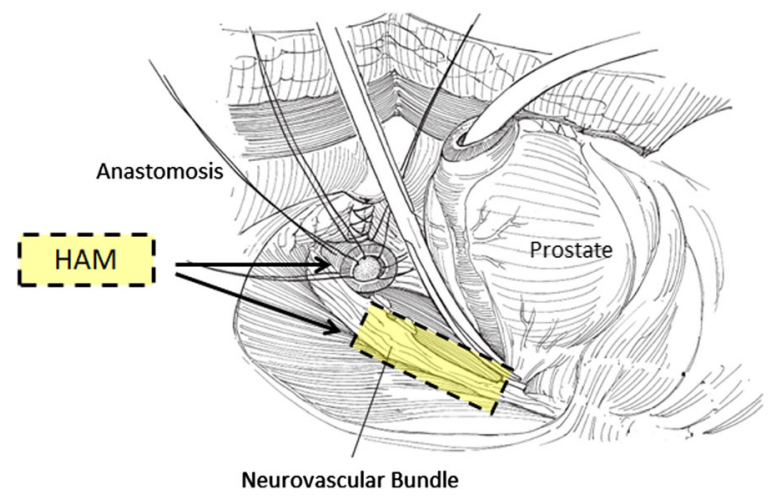

Fig. 1 Surgical procedure of radical retropubic prostatectomy. Dehydrated HAM is cut in three pieces and placed over the neurovascular bundles and at a later stage of the surgery wrapped around the sutured vesicourethral anastomosis (adopted from Hinman's Atlas of Urologic Surgery) [27]

which go beyond the requirements of the $\mathrm{EU}$ Directive 2006/17, and the application of a validated, chemical, cold sterilization process, the transmission of infectious diseases by previously unknown or undetectable pathogens cannot be ruled out with complete certainty. The graft can be applied immediately to the repair site without rehydration.

\section{Protocol Development}

A protocol for a prospective single-blinded, randomized, placebo-controlled phase 2 study to improve post-prostatectomy incontinence and potency by application of dried human amnion graft has been developed in collaboration with the participating institutions. The protocol has been created according to the CONSORT recommendations (http://www. consort-statement.org). It has been evaluated and adapted to the SPIRIT (Standard Protocol Items Recommendations for Interventional Trials) 2013 Checklist (http://www.spiritstatement.org/wp-content/uploads/2013/01/SPI RIT-Checklist-download-8Jan13.pdf). A CONSORT diagram is provided for the work flow of this clinical trial (Fig. 2).

This trial is designed in accordance with the Standard Protocol Items for Clinical Trials (SPIRIT) 2013 statement and will be carried out in compliance with the ethical principles of the Declaration of Helsinki. The protocol will be approved by the regional ethical commissions of the participating center and possible additional centers. The study will only start once those institutions have given their positive vote. This article does not contain any new 


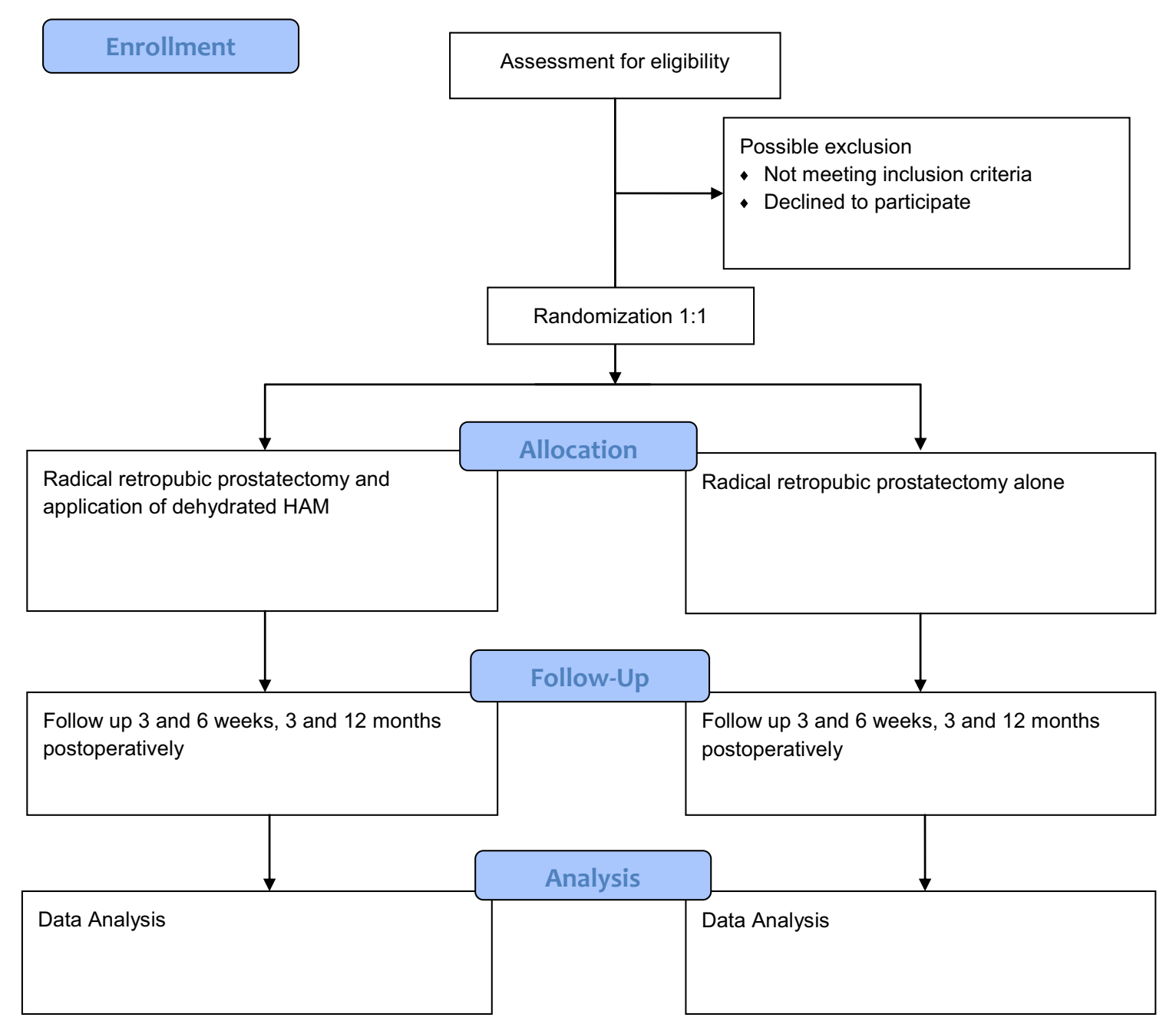

Fig. 2 CONSORT flow diagram for current trial

studies with human or animal subjects performed by any of the authors.

\section{Study Population}

Eligible men aged 40-80 years with localized prostate cancer, indication for and choice of radical prostatectomy treatment will be identified by their treating office-based urologists and referred to the study center, Rhineland Hospital Neuss, Germany. Eligible men who declare an interest in participating in the trial will receive a patient information leaflet describing the study and informing them about the scientific background, previous results, etc. They will also be supplied with specific questionnaires and a consent form as well as an information sheet concerning the respective study participation insurance. All patients will sign an informed consent for randomization and in order to use their data for the study. Once those documents are received, every men will get a personal study number.

(a) Inclusion criteria: male adults of 40-80 years old, with localized prostate cancer, indication for radical prostatectomy, no other treatment of prostate cancer, availability to sign informed consent.

(b) Exclusion criteria: preoperative incontinence (24-h pad test), preoperative erectile dysfunction (IIEF-5 $<20$ ), metastasized or locally advanced prostate cancer in 
preoperative assessment, previous radiation of pelvis, previous prostate cancer therapy, psychiatric disease (excluding depression and anxiety if stable condition), participation in another study, reduced compliance and inability to complete at least 12 months of follow-up (due to long distance to the hospital or work issues).

\section{Planned Outcomes and Measurements}

\section{Outcome Measurement}

Urinary incontinence over $24 \mathrm{~h}$ will be assessed by pad weighing test according to ICS standard [28]. Pad tests are used for the quantitative measurement of involuntary loss of urine. The pad is weighed before and again after $24 \mathrm{~h}$. The difference in weights gives the measurement of urine loss in grams. If a pad is insufficient for $24 \mathrm{~h}$, the weight of the pad is weighed and noted for $24 \mathrm{~h}$ after each change. The following classification of incontinence is performed: very strong incontinence, more than $450 \mathrm{~g}$ in $24 \mathrm{~h}$; strong incontinence, $200-450 \mathrm{~g}$ in $24 \mathrm{~h}$; medium-grade incontinence, 50-200 g in $24 \mathrm{~h}$; light incontinence, less than $50 \mathrm{~g}$ in $24 \mathrm{~h}$; complete continence, less than 2-3 g in $24 \mathrm{~h}$. Decision to remove the catheter after the RP is made on the basis of cystogram on 5-7 postoperative day (instillation of $200 \mathrm{ml}$ iodinated contrast agent) and examination of anastomosis for leaks in two planes. The radiological result is documented in the picture as well as in writing. In case of contrast agent leakage, the catheter is left and the procedure will be repeated after 1 week. Catheter removal is reported in number of days postoperatively. Potency will be assessed using the validated IIEF-5 questionnaire [29]. IIEF-5 of 17 points or fewer is defined as moderate to severe erectile dysfunction. Biochemical recurrence is assessed on the basis of the prostate-specific antigen (PSA) value postoperatively. The biochemical recurrence after radical prostatectomy is defined by PSA concentrations above $0.2 \mathrm{ng} / \mathrm{ml}$ more than 3 months after prostatectomy, which are increasing in two controls. The cause may be local recurrence or distant metastases [30]. Complications are assessed by Clavien-Dindo classification [31].

\section{Primary Outcome Measures}

Change in urine loss will be objectified by $24-\mathrm{h}$ pad-test (in grams). The time frame will be from baseline (1 week after surgery) to the rehabilitation training (3-6 weeks after surgery) and to 3 and 12 months after surgery.

\section{Secondary Outcome Measures}

1. Change in erectile function (EF) will be assessed by IIEF- 5 questionnaire. The time frame will be from baseline (4 weeks before surgery) to the rehabilitation training (3-6 weeks after surgery) and to 3 and 12 months after surgery.

2. Evaluation of the catheter residence time as a parameter of internal wound healing of VUA will be objectified by the time of postoperative catheter removal (days).

3. Complications will be assessed according to Clavien-Dindo classification perioperatively, at 6 weeks, 3 months, and 12 months.

4. Biochemical recurrence will be objectified by the measurement of PSA at 6 weeks, 3 months, and 12 months.

\section{Randomization and Blinding}

This study cannot be double-blind since the application of HAM is performed during the surgical procedure by the surgeon. Therefore the study is single-blind to the patient. A 1:1 randomization is chosen; allocation into the two study groups will be completely randomized using the program http://www.randomizer. at. This randomization application will be available as an Internet-based service. Randomization will be carried out as close to the time of surgery as is possible.

\section{Data Analysis and Sample Size Calculation}

The main objective of the study is to reduce the 24-h urine loss on the first day of rehab training. In both groups the same distribution with the same standard deviation is assumed. On the basis of clinical experience (own data from the years 2016-2018), the 24-h pad weighing test on the first day of rehab training can be based on a mean of $80 \mathrm{ml}$ and a standard deviation of $115 \mathrm{ml}$. Patients with urine loss in excess of 
$400 \mathrm{ml}$ were excluded from the study because no improvement due to amnion application is expected. HAM application is considered effective if the mean assumed 24-h urine loss is $40 \mathrm{ml}$ at the same standard deviation. For evaluation, the two-tailed two-sample $t$ test will be used at a significance level of $\alpha=0.05$. With a test power of $80 \%, 131$ patients are required, and with a $20 \%$ drop ut rate expected, 164 patients per group are required. With the recruitment of five patients per month, the recruitment phase is expected to be 66 months. Patients are all monitored over a 12-month period as part of regular medical follow-up and according to the study objectives.

\section{Data Collection}

Documentation of protocol-required information will be performed via standardized case report forms which will be reviewed and signed by the investigator or an appropriate subinvestigator. All data will be transferred pseudonymously. Patients and research staff will not be explicitly informed if HAM was applied or not during the surgical procedure. It will not be possible to get this information via examination features, etc., either for the patient or for the physician involved in the follow-up. The data important for the clinical trial will be stored digitally and in paper form, evaluated and archived in pseudonymized form. Data collection and archiving are carried out taking into account the legal data protection regulations and retention periods. Transmission of data will be done in an encrypted format. No access to the original documents will be given to others not involved in the trial. Both patient and physician will sign the informed consent to obtain and validate it. Participation in this study is voluntary and revocable at any time without disclosure of the motives. In this case all relevant data will be deleted if desired by the patient.

\section{DISCUSSION}

Only very few studies are available on postprostatectomy incontinence. Stage 0-2 IDEAL studies were revealed by systematic review of the literature [32].

Heesakkers et al. recently published a review on intraoperative surgical factors contributing to incontinence after surgery, which shows that the level of evidence of most trials is low [33]. The studies in this review showed that stricture formation, extensive dissection, neurovascular bundle damage, devascularization, and fibrosis were negative predictive factors of postoperative incontinence. There is debate about the role and extent of the contribution of the NVB in the innervations of the external urethral rhabdosphincter and membraneous urethra [34, 35].

Strasser and Bartsch studied 19 male cadavers to clarify the anatomy of pelvis and especially urethra innervation. The protection of nervi cavernosi and the erectile nerves in the field of seminal vesicles of the prostate and in the area of the apical urethra are crucial for nerve-sparing pelvic surgery in man. Nerve preservation take place by preservation of the seminal vesicles and neurovascular bundles in the presented protocol. However, it is outmost important to preserve the fine branches of the nervus pudendus, which innervate the rhabdosphincter and the surrounding muscle and vascular structures, by careful apical dissection of the urethra [35].

The cellular environment of an injured urinary tract wall gradually becomes highly cytotoxic as a result of traumatization of the surrounding vascular plexus and in consequence related hypoxia. These factors are responsible for the chronic surgical reconstruction failures of the urinary tract whose developmental risk is somewhat independent of applied surgery techniques but is associated instead with a natural sequence of the healing process. A fast healing of the muscle and fine nerve branches in this area would lead to a better functional outcome and early recovery of continency and potency after RP. This has been shown by several studies (IDEAL stage $1-2 b$ ) $[36,37]$.

The afferent innervation and its effect on subsequent urinary continence are even less well understood. Catarin et al. evaluated urethral afferent activity, which appeared to be 
disrupted after RP and could have an influence on continence after RP (IDEAL stage 2) [38].

Against the background of extended preliminary animal trials we hypothesized that HAM application to neurovascular bundles and vesicourethral anastomosis during the radical prostatectomy could reduce local inflammation, nerve and muscle scarring. It should therefore result in the early return to continence and potency.

One of the possible biases is that patients with and without NVB-sparing surgery will be included. However, we assume that the preservation of the nerve plexus on the dorsal site of the prostate, the fine afferent and efferent branches of nervus pudendus, and the preservation of vascularization of the urethra by HAM application would play an important role for continence recovery.

The retrospective data on the use of dehydrated human amnion (AmnioFix, MiMedx, Marietta, GA, USA) as part of a nerve-sparing robotic RP showed a faster return to potency and continence with no increased risk of relapse compared to a matched control group of 58 patients during nerve-sparing robotic assisted RP (IDEAL stage 2a) [39]. Mean time to continence was enhanced in HAM-group patients (1.21 months) versus (1.83 months; $p=0.033)$ and mean time to potency was 1.34 months versus 3.39 months; IIEF-5 score was higher in the HAM group 16.2 vs. 9.1. Another group matched 1400 patients undergoing full bilateral nerve-sparing robotic assisted RP by a single surgeon, wherein 700 patients had dHAM allograft wrapped around the NVB and 700 did not with retrospective follow-up of 1 year (IDEAL stage 2a) [40]. Groups were matched demographically, clinically, and biochemically. Binary logistic regression demonstrated that HAM application was an independent significant $(p<0.001)$ predictor of achieving potency at 1 year, such that the patients with HAM were 3.86 times (95\% CI 2.43-6.13) more likely to achieve potency in the same period when compared with control group. As a result of its extracellular matrix composition, HAM is assumed to be preferable for tissue outgrowth. Amnion provided faster healing of vesicourethral anastomosis and reduced indwelling catheter time in a small retrospective study for 15 patients during salvage RP (IDEAL stage 1) [26]. The time of catheter removal was an independent predictor of urinary continence recovery after RP in a prospective trial with 197 patients (IDEAL stage 2a) [41]. HAM could be a suitable scaffold for faster and improved VUA healing and therefore continence recovery.

Several studies have indicated that HAM might display anticancer activity. A suppression of different cancer lines by the addition of HAM in vitro has been shown, but clinical studies are missing [42, 43]. In the presented protocol oncologic results are being recorded to assess the biochemical recurrence. However, longer follow-up is required to evaluate future outcomes for our cohort.

This study protocol presents the first prospective randomized exploration study with larger sample sizes to prove this novel approach.

\section{STRENGTHS AND LIMITATIONS}

Our entire preliminary experimental approach has been strictly performed following the IDEAL recommendations for surgical innovations. Besides the animal studies, the method of HAM application in the urinary tract has already been proven feasible in case reports and several propensity score matched retrospective studies for RP. Thus, according to IDEAL, the method needs to be assessed, most preferably in a randomized controlled trial setting. However, a big effort with a large number of patients and structured follow-up is needed. Another strength is that this trial can be easily randomized and single-blinded to the patient. Previous data show that there is no increased risk when applying HAM, with no graft rejection to be expected. One study center and one surgeon will ensure the outcome reproducibility. As the procedure is done by a highly experienced surgeon and amnion application is simple so no learning curve needs to be included. Outcome assessment is achieved via participant selfcompleted questionnaire and standardized 24-h pad test, which prevents interviewer bias.

This study protocol has some limitations. An important limitation is the large number of 
required patients ( $n=131$, not including calculated lost to follow-up patients) per randomization arm which is necessary to prove the main outcome goal of early incontinence reduction. As this study is monocentric in nature and the center is a high volume institution, recruiting 328 patients over 5 years is likely to be difficult. As only one surgeon will perform the procedures, this can be vulnerable if he is not available for a shorter or longer period. However, inclusion of additional surgeons and centers by amendment is possible according to the protocol. According to the IDEAL recommendations it is important to get a stable procedure and identify risks and iterations needed before it can be used by other adopters. Another limiting factor for the success of this trial is the challenge of finding appropriate funding sources or even financial supporters for a randomized trial with already approved medicament. Human amniotic tissue is approved according to Pharmaceutical German Law and is therefore strictly regulated. On the contrary, several commercial grafts are available in the USA and are less regulated as a human cell, tissue, or cellular or tissue-based product (HCT/P) under Section 361 of the Public Health Service Act. However, after successful publication of an approved trial protocol, institution funds and public funds will be targeted. In this particular project it is of outmost importance that all IDEAL stages are covered and published accordingly, in order to evaluate the potential of HAM in broad clinical application.

\section{ACKNOWLEDGEMENTS}

Funding. No funding or sponsorship was received for this study or publication of this article. Potential institutions will be addressed subsequently to publishing the protocol.

Authorship. All named authors meet the International Committee of Medical Journal Editors (ICMJE) criteria for authorship for this article, take responsibility for the integrity of the work as a whole, and have given final approval for this version to be published.
Authorship Contributions. DB, HG, TE, MB, JB, IT, AR, UB and TO contributed to the conception and design of the study and developed the study protocol. DB and TO are responsible for the recruitment of subjects. DB, HG, UB and $\mathrm{TE}$ are responsible for the management of the trial, collection and analysis of the data. All authors contributed to modification of the original protocol and all authors read and approved the final manuscript.

Disclosures. Dimitri Barski, Holger Gerullis, Thorsten Ecke, Mihaly Boros, Jan Brune, Ulrich Beutner, Igor Tsaur, Albert Ramon and Thomas Otto have nothing to disclose.

Compliance with Ethics Guidelines. This trial is designed in accordance with the Standard Protocol Items for Clinical Trials (SPIRIT) 2013 statement and will be carried out in compliance with the ethical principles of the Declaration of Helsinki. The protocol will be approved by the regional ethical commissions of the participating center and possible additional centers. The study will only start once those institutions have given their positive vote. This article does not contain any new studies with human or animal subjects performed by any of the authors.

Data Availability. The datasets generated during and/or analyzed during the current study are available from the corresponding author on reasonable request.

Open Access. This article is distributed under the terms of the Creative Commons Attribution-NonCommercial 4.0 International License (http://creativecommons.org/licenses/ by-nc/4.0/), which permits any noncommercial use, distribution, and reproduction in any medium, provided you give appropriate credit to the original author(s) and the source, provide a link to the Creative Commons license, and indicate if changes were made. 


\section{REFERENCES}

1. Ficarra V, Novara G, Rosen RC, et al. Systematic review and meta-analysis of studies reporting urinary continence recovery after robot-assisted radical prostatectomy. Eur Urol. 2012;62(3):405-17.

2. Ellison JS, He C, Wood DP. Stratification of postprostatectomy urinary function using expanded prostate cancer index composite. Urology. 2013;81(1):56-60.

3. Capogrosso P, Salonia A, Briganti A, Montorsi F. Postprostatectomy erectile dysfunction: a review. World J Mens Health. 2016;34(2):73-88.

4. Lepor H, Kaci L, Xue X. Continence following radical retropubic prostatectomy using self-reporting instruments. J Urol. 2004;171(3):1212-5.

5. O'Sullivan R, Karantanis E, Stevermuer TL, Allen W, Moore KH. Definition of mild, moderate and severe incontinence on the $24-\mathrm{h}$ pad test. BJOG. 2004;111(8):859-62.

6. Asimakopoulos AD, Miano R, Galfano A, et al. Retzius-sparing robot-assisted laparoscopic radical prostatectomy: critical appraisal of the anatomic landmarks for a complete intrafascial approach. Clin Anat. 2015;28(7):896-902.

7. Coughlin GD, Yaxley JW, Chambers SK, et al. Robot-assisted laparoscopic prostatectomy versus open radical retropubic prostatectomy: 24-month outcomes from a randomised controlled study. Lancet Oncol. 2018;19(8):1051-60.

8. Ilic D, Evans SM, Allan CA, Jung JH, Murphy D, Frydenberg M. Laparoscopic and robotic-assisted versus open radical prostatectomy for the treatment of localised prostate cancer. Cochrane Database Syst Rev. 2017;9:CD009625.

9. Lane JM, Bora FW, Pleasure D. Neuroma scar formation in rats following peripheral nerve transection. J Bone Jt Surg Am. 1978;60(2):197-203.

10. Menon M, Kaul S, Bhandari A, Shrivastava A, Tewari A, Hemal A. Potency following robotic radical prostatectomy: a questionnaire based analysis of outcomes after conventional nerve sparing and prostatic fascia sparing techniques. J Urol. 2005;174(6):2291-6 (discussion 6).

11. Ogaya-Pinies G, Palayapalam-Ganapathi H, Rogers $\mathrm{T}$, et al. Can dehydrated human amnion/chorion membrane accelerate the return to potency after a nerve-sparing robotic-assisted radical prostatectomy? Propensity score-matched analysis. J Robot Surg. 2018;12(2):235-43.
12. Ricci E, Vanosi G, Lindenmair A, et al. Anti-fibrotic effects of fresh and cryopreserved human amniotic membrane in a rat liver fibrosis model. Cell Tissue Bank. 2013;14(3):475-88.

13. Fetterolf DE, Snyder RJ. Scientific and clinical support for the use of dehydrated amniotic membrane in wound management. Wounds. 2012;24(10): 299-307.

14. Lemke A, Ferguson J, Gross K, et al. Transplantation of human amnion prevents recurring adhesions and ameliorates fibrosis in a rat model of sciatic nerve scarring. Acta Biomater. 2018;66:335-49.

15. Ilancheran S, Michalska A, Peh G, Wallace EM, Pera M, Manuelpillai U. Stem cells derived from human fetal membranes display multilineage differentiation potential. Biol Reprod. 2007;77(3):577-88.

16. Kim JC, Tseng SC. Transplantation of preserved human amniotic membrane for surface reconstruction in severely damaged rabbit corneas. Cornea. $1995 ; 14(5): 473-84$.

17. Zelen CM, Serena TE, Denoziere G, Fetterolf DE. A prospective randomised comparative parallel study of amniotic membrane wound graft in the management of diabetic foot ulcers. Int Wound J. 2013;10(5):502-7.

18. Barski D, Gerullis H, Ecke T, et al. Human amniotic membrane is not suitable for the grafting of colon lesions and prevention of adhesions in a xenograft rat model. Surg Innov. 2017;24(4):313-20.

19. Barski D, Gerullis H, Ecke T, et al. Human amniotic membrane dressing for the treatment of an infected wound due to an entero-cutaneous fistula: case report. Int J Surg Case Rep. 2018;51:11-3.

20. Barski D, Gerullis H, Ecke T, et al. Repair of a vesicovaginal fistula with amniotic membrane-step 1 of the IDEAL recommendations of surgical innovation. Cent Eur J Urol. 2015;68(4):459-61.

21. Barski D, Gerullis H, Ecke T, et al. Bladder reconstruction with human amniotic membrane in a xenograft rat model: a preclinical study. Int J Med Sci. 2017;14(4):310-8.

22. McCulloch P, Altman DG, Campbell WB, et al. No surgical innovation without evaluation: the IDEAL recommendations. Lancet. 2009;374(9695): 1105-12.

23. Sedrakyan A, Campbell B, Merino JG, Kuntz R, Hirst A, McCulloch P. IDEAL-D: a rational framework for evaluating and regulating the use of medical devices. BMJ. 2016;353:i2372. 
24. Walsh PC. Re: anatomical radical retropubic prostatectomy: detailed description of the surgical technique. J Urol. 2005;173(1):324.

25. Schäfers S, de Geeter P, Löhmer H, Albers P. Seminal vesicle sparing radical perineal prostatectomy. Urologe A. 2009;48(4):408-14.

26. Ogaya-Pinies G, Kadakia Y, Palayapalayam-Ganapathi $\mathrm{H}$, et al. Use of scaffolding tissue biografts to bolster vesicourethral anastomosis during salvage robot-assisted prostatectomy reduces leak rates and catheter times. Eur Urol. 2018;74(1):92-8.

27. Smith JA, Howards SS, Preminger GM, Hinman F. Hinman's atlas of urologic surgery. 3rd ed. Philadelphia: Elsevier; 2012. p. 408.

28. Abrams P, Cardozo L, Khoury S, Wein A. 2005. Incontinence basics \& evaluation, 3rd International consultation on incontinence. Chapter 13: Imaging and other Investigations. http://www.icsoffice.org/. Accessed 3 Mar 2019.

29. Rosen RC, Riley A, Wagner G, Osterloh IH, Kirkpatrick J, Mishra A. The international index of erectile function (IIEF): a multidimensional scale for assessment of erectile dysfunction. Urology. 1997;49(6):822-30.

30. Mottet N, Bellmunt J, Bolla M, et al. EAU-ESTROSIOG guidelines on prostate cancer. Part 1: screening, diagnosis, and local treatment with curative intent. Eur Urol. 2017;71(4):618-29.

31. Dindo D, Demartines N, Clavien PA. Classification of surgical complications: a new proposal with evaluation in a cohort of 6336 patients and results of a survey. Ann Surg. 2004;240(2):205-13.

32. Barski D, Gerullis H, Otto T. Review of surgical implant procedures for male incontinence after radical prostatectomy according to IDEAL framework. Updates Surg. 2017;69(3):327-38.

33. Heesakkers J, Farag F, Bauer RM, Sandhu J, De Ridder D, Stenzl A. Pathophysiology and contributing factors in postprostatectomy incontinence: a review. Eur Urol. 2017;71(6):936-44.

34. Gosling JA, Dixon JS. The structure and innervation of smooth muscle in the wall of the bladder neck and proximal urethra. Br J Urol. 1975;47(5):549-58.
35. Strasser H, Bartsch G. Anatomic basis for the innervation of the male pelvis. Urologe A. 2004;43(2):128-32.

36. Ozdemir MB, Eskicorapci SY, Baydar DE, Cumhur M, Onderoğlu S, Ozen H. A cadaveric histological investigation of the prostate with three-dimensional reconstruction for better results in continence and erectile function after radical prostatectomy. Prostate Cancer Prostatic Dis. 2007;10(1):77-81.

37. Kaye DR, Hyndman ME, Segal RL, et al. Urinary outcomes are significantly affected by nerve sparing quality during radical prostatectomy. Urology. 2013;82(6):1348-53.

38. Catarin MV, Manzano GM, Nóbrega JA, Almeida FG, Srougi M, Bruschini H. The role of membranous urethral afferent autonomic innervation in the continence mechanism after nerve sparing radical prostatectomy: a clinical and prospective study. J Urol. 2008;180(6):2527-31.

39. Patel VR, Samavedi S, Bates AS, et al. Dehydrated human amnion/chorion membrane allograft nerve wrap around the prostatic neurovascular bundle accelerates early return to continence and potency following robot-assisted radical prostatectomy: propensity score-matched analysis. Eur Urol. 2015;67(6):977-80.

40. Razdan S, Bajpai RR, Sanchez MA. A matched and controlled longitudinal cohort study of dehydrated human amniotic membrane allograft sheet used as a wraparound nerve bundles in robotic-assisted laparoscopic radical prostatectomy: a puissant adjunct for enhanced potency outcomes. J Robot Surg. 2019;13(3):475-81.

41. Rossanese M, Crestani A, Palumbo V, et al. Time of catheterization as an independent predictor of early urinary continence recovery after radical prostatectomy. Minerva Urol Nefrol. 2018;70(4):401-7.

42. Niknejad H, Khayat-Khoei M, Peirovi H, Abolghasemi H. Human amniotic epithelial cells induce apoptosis of cancer cells: a new anti-tumor therapeutic strategy. Cytotherapy. 2014;16(1):33-40.

43. Bu S, Zhang Q, Wang Q, Lai D. Human amniotic epithelial cells inhibit growth of epithelial ovarian cancer cells via TGF $\beta 1$-mediated cell cycle arrest. Int J Oncol. 2017;51(5):1405-14. 